included training-related injuries. Different study designs, injury and illness definitions, race distances, and surfaces, made pooling of results difficult. The foot, knee, ankle and thigh are the most common anatomical sites of TR injury, with lacerations/abrasions, blisters, muscle strains, cramping and ankle sprains most commonly diagnosed. TR illness involved the gastrointestinal tract (GIT), metabolic and cardiovascular body systems. Symptoms of nausea and vomiting related to GIT distress and dehydration are commonly reported.

Conclusions Injury and illness are common among TRs participating in TR races. Limited evidence is available on trainingrelated injury and illness in TR specific.

\section{RELATIONSHIP OF PATELLOFEMORAL ANGLES AND TIBIOFEMORAL ROTATIONAL ANGLES WITH JUMPER'S KNEE IN PROFESSIONAL FOLK DANCERS: AN MRI ANALYSIS}

${ }^{1}$ Neslihan Aksu, ${ }^{1}$ Vefa Atansay, ${ }^{2}$ ssik Karalok, ${ }^{1}$ Ayhan Nedim Kara, ${ }^{3}$ Azmi Hamzaoglu. ${ }^{1}$ Demiroglu Bilim University Medical Faculty Florence Nightingale Hospital Orthopedics and Traumatology Department, Istanbul, Turkey; ${ }^{2}$ Demiroglu Bilim University Medical Faculty Florence Nightingale Hospital Radiology Department, Istanbul, Turkey; ${ }^{3}$ Istanbul Florence Nightingale Hospital Orthopedic and Spine Center, Istanbul, Turkey

\subsection{6/bjsports-2021-IOC.305}

Background Professional dancers learn splash and landing techniques throughout their careers starting in childhood and practice it very frequently like basketball, volleyball and soccer. Among the intrinsic factors, anatomical features of the lower extremity were the most studied in the literature.

Objective In this article, we investigated the relationship of tibiofemoral rotational angles and patellofemoral (PF) angles to the development of jumper's knee in professional folk dancers.

Design Retrospective cohort MRI study.

Setting Professional folk dance group.

Patients (or Participants) 26 professional folk dancers (16 male, 10 female; mean age of $30.69 \pm 7.51$ years (17 to 46$)$ ) group with complaints of knee pain.

Interventions (or Assessment of Risk Factors) PF sulcus angle and Femur-Insall angle were found to be related to Jumper's knee.

Main Outcome Measurements We examined 26 dancers with complaints of knee pain, and 32 knees of them had magnetic resonance imaging (MRI). We detected 21 jumper's knees. We measured patellofemoral angles (Patellofemoral sulcus angle, Lateral patellofemoral angle, Patellar tilt angle, Lateral trochlear-inclination angle, Lateral patellar tilt angle, The patellofemoral congruence angle) and tibiofemoral rotational angles (Condillary twist angles, posterior condillary angles, femurInsall angles, tibia-Insall angles, posterior tibiofemoral angles, Whiteside-PFCL angles) and noted patellar specifics as alta, Baja, Wiberg on MRI's with and without jumper's knee to understand if there is any relationship with tendinopathy occurrence in this cohort study.

Results According to logistic regression analysis, PF sulcus angle was found to be related to quadriceps tendinopathy development $(\mathrm{p}<0,05$, odd ratio (OR): 1,24, 95\% confidential interval (CI ): 1,03-1,5) and patellar tendinopathy is found to be related to Femur-Insall angle $(\mathrm{p}<0,05$, OR: 1,27, 95\% CI: $1,00-1,61)$.
Conclusions The patellofemoral sulcus angle and patellar tendon rotation relative to the femur may be the effective anatomical variations in jumper's knee occurence.

\section{CALCANEAL BONE DENSITY AND BONE STRESS INJURY IN NCAA DIVISION I ATHLETES AND NON- INTERCOLLEGIATE ATHLETE COLLEGE STUDENTS}

${ }^{1}$ Jason Bennett, ${ }^{2}$ Tricia Austin, ${ }^{2}$ Ann Hayes, ${ }^{3}$ Mark Reinking. ${ }^{1}$ Carroll University, Waukesha, USA; ${ }^{2}$ Saint Louis University, Saint Louis, USA; ${ }^{3}$ Regis University, Denver, USA

\subsection{6/bjsports-2021-IOC.306}

Background There is limited evidence describing the relationship between calcaneal bone mineral density (cBMD) and activity level or lower extremity overuse bone injury (LEOBI). Objective The purposes of this study were to: 1) compare cBMD of intercollegiate athletes (ICA) and non-intercollegiate athlete (NA) college students, 2) examine the influence of physical activity on cBMD in NA, and 3) determine if there is an association between CBMD and the development of LEOBI.

Design Prospective, cohort study.

Setting NCAA Division I University.

Participants 84 ICA and 103 NA college students.

Assessment ICAs provided injury and menstrual history, were measured for $\mathrm{CBMD}$ at the beginning and end of the year, and were followed for occurrence of LEOBI. NA college students provided injury and menstrual history and were measured for cBMD.

Main Outcome Measures Descriptive statistics, statistical analyses of relationships, logistic regression, and t-tests were used in the statistical analyses.

Results Eight ICAs were diagnosed with a LEOBI over the year. There was no difference in initial cBMD between ICAs with and without LEOBI; right $(\mathrm{p}=.05)$ and left $\mathrm{cBMD}$ $(p=.07)$ were lower in those ICAs with LEOBI at the end of the season. The NAs had significantly lower cBMD and speed of sound (SOS) than the ICAs. There were no significant differences in $\mathrm{cBMD}$ and SOS values between the 8 ICAs with LEOBI and the 103 NAs. For the NAs, there was no significant correlation between cBMD and activity, however, age of onset of menstruation and CBMD were found to be significantly correlated $(\mathrm{p}<.05)$.

Conclusions cBMD was significantly lower in NAs as compared to ICAs. The ICAs with LEOBI did not have significantly different cBMD than the NAs. The difference in cBMD between ICAs and NAs may be activity related, but differences in cBMD among the NAs was not related to activity level.

\section{HIP EXTENSOR WEAKNESS IS ASSOCIATED WITH LOWER LIMB MUSCLE STRAIN IN MALE ELITE VOLLEYBALL ATHLETES}

${ }^{1}$ Natália FN Bittencourt, ${ }^{1}$ Renato de Paula da Silva, ${ }^{1}$ Paola de Figueiredo Caldeira, ${ }^{2}$ Alysson Lima Zuin, ${ }^{2}$ Daniel Bornelli Campos Serio, ${ }^{2}$ Petterson Moura da Silva, ${ }^{1,3}$ Luciana De Michelis Mendonça. 'PHAST - Physiotherapy Assessment Tool, Belo Horizonte, Brazil; ${ }^{2}$ Sada Cruzeiro Vôlei, Belo Horizonte, Brazil; 3UFVIM - Universidade Federal dos Vales do Jequitinhonha e Mucuri, Diamantina, Brazil

10.1136/bjsports-2021-IOC.307 